FINAL REPORT

\title{
EFFECT OF ELECTRONIC EXCITATION ON THIN FILM GROWTH
}

Period Covered

1 February 2009 - 31 January 2011

97ER45625

\author{
Hani E. Elsayed-Ali (P.I.) \\ Professor \\ Department of Electrical and Computer Engineering \\ Old Dominion University \\ Norfolk, Virginia 23529-0246
}

May 2013

Prepared for

The U.S. Department of Energy

This report was prepared as an account of work sponsored by the United States Government. Neither the United States nor the Department of Energy, nor any of their employees, nor any of their contractors, subcontractors, or their employees, makes any warranty, express or implied, or assumes any legal liability or responsibility for the accuracy, completeness, or usefulness of any information, apparatus, product or process disclosed or represents that its use would not infringe privately-owned rights. 


\section{Refereed Publications Based on DOE Funding}

Ahmed R. Esmail, Aleksey Bugayev, and Hani E. Elsayled-Ali, "Electron diffraction studies of structural dynamics of bismuth nanoparticles," J. Phys. Chem. C 117 (17), 9035-9041 (2013).

M. A. Hafez, M. A. Mamun, A. A. Elmustafa, and H. E. Elsayed-Ali, "Structural and nanomechanical properties of InN films grown on $\mathrm{Si}(100)$ by femtosecond pulsed laser deposition," J. Phys. D: Appl. Phys. 46, 175301 (8pp) (2013).

Ashraf Hassan Farha, Ali Oguz Er, Yuksel Ufuktepe, Ganapati Myneni, and Hani E. Elsayed-Ali, "Effects of substrate temperature on properties of $\mathrm{NbNx}$ films grown on $\mathrm{Nb}$ by pulsed laser deposition," Appl. Surf. Sci. 258, 1613-1618 (2011). (Partial Funding)

Ahmed R. Esmail and Hani E. Elsayed-Ali, "Anisotropic response of nanosized bismuth film upon femtosecond laser excitation monitored by ultrafast electron diffraction," Appl. Phys. Lett. 99, 161905 (3 pages) (2011).

Ashraf Hassan Farha, Ali Oguz Er, Yuksel Ufuktepe, Ganapati Myneni, and Hani E. Elsayed-Ali, "Influence of nitrogen background pressure on structure of niobium nitride films grown by pulsed laser deposition," Surface \& Coatings Technology 206, 1168-1174 (2011). (Partial funding)

Ali Oguz Er and Hani E. Elsayed-Ali, "Femtosecond pulsed laser deposition of Ge quantum dot growth on Si(1 0 0)-(2 × 1)," Appl. Surf. Sci. 257, 8078-8084 (2011).

A. Esmail, M. Abdel-Fattah, and H. E. Elsayed-Ali, "Nonuniformity in lattice contraction of bismuth nanoclusters heated near its melting point," J. Appl. Phys. 109, 084317 (6 pages) (2011).

A. Oguz Er and H. E. Elsayed-Ali, "Electronically Enhanced Surface Diffusion during Ge Growth on Si(100)," J. Appl. Phys. 109, 084320 (6 pages) (2011).

A. Bugayev, A. Esmail, M. Abdel-Fattah, and H. E. Elsayed-Ali, "Coherent phonons in bismuth film observed by ultrafast electron diffraction," AIP Advances 1, 012117 (5 pages) (2011).

$\left(3^{\text {rd }}\right.$ most downloaded article from AIP Advances in April 2011, top 20 downloaded March-May, 2011)

A. Oguz Er, Wei Ren, and H. E. Elsayed-Ali, "Low temperature epitaxial growth of Ge quantum dot on Si(100)-(2x1) by femtosecond laser excitation," Appl. Phys. Lett. 98, 013108 (3 pages) (2011); selected for publication in Virtual Journal of Ultrafast Science, February (2011).

A. Oguz Er and H. E. Elsayed-Ali, "Excitation induced Ge quantum dot formation on Si(100)-(2x1)," J. Appl. Phys. 108, 034303 (10 pages) (2010).

M. A. Hafez and H. E. Elsayed-Ali, "Low-temperature growth of InN on $\mathrm{Si}(100)$ by femtosecond pulsed laser deposition," J. Vac. Sci. \& Technol. A 27(4), 696-699 (2009). Also, selected for publication in the Virtual Journal of Ultrafast Science 8 (7) (2009).

\section{Invited and Plenary Talks}

(Invited): H. E. Elsayed-Ali, "Coherent phonons in polycrystalline bismuth film monitored by ultrafast electron diffraction," $1^{\text {st }}$ International Conference on phononic Crystals, Metamaterials, and Optomechanics (Phononics 2011), p. 210-211, May 29-June 2, 2011.

(Invited): H. E. Elsayed-Ali, "Ultrafast structural dynamics of surfaces and thin films studied by electron diffraction," Symposium on Dynamic Transmission Electron Microscopy, INRS, University of Quebec, Montreal, Canada, May 9-11, 2011. 
(Plenary talk): H. E. Elsayed-Ali, "Measurements of heat transport in thin films by ultrafast laser-based techniques," Third International Conference on Thermal Issues in Emerging Technologies Theory and Application - ThETA 3, Cairo, Egypt, December 19-22, 2010.

\section{Program Scientists and Graduate Students}

Dr. H. E. Elsayed-Ali, P.I.

M. Abdel-Fattah, "Ultrafast Diffraction Studies of Antimony Thin Films and Nanoparticles," Ph.D. dissertation, Department of Electrical and Computer Engineering, Old Dominion University, August 2011.

A. Oguz Er, "Excitation-induced Ge Quantum Dot Formation on Si(100)-2x1 by Pulsed Laser Deposition," Ph.D. dissertation, Department of Physics, Old Dominion University, August 2011.

A. Esmail, "Ultrafast high-energy electron diffraction Study of Photoexcited Bismuth Nanoclusters by Femtosecond Laser pulses," Ph.D. dissertation, Department of Electrical and Computer Engineering, Old Dominion University, August 2011.

I. El-Kholy, "Surface Dynamics of Si Low-Index Surfaces Studied by Reflection High Energy Electron Diffraction," Ph.D. dissertation, Department of Electrical and Computer Engineering, Old Dominion University, May 2009.

Wei Ren, $4^{\text {nd }}$ year grad student, Ph.D. candidate, "Ultrafast Electron Diffraction Studies of Quantum Size effect in Germanium," in progress

\section{Research Findings}

The research findings focuses on two areas: (A) Effect of electronic excitation applied by ultrafast lasers on thin film growth, (B) Ultrafast electron diffraction studies of femtosecond laser excitation of semimetals (Bi and $\mathrm{Sn}$ ). The results are summarized below and can be found in the referenced publications:

(A) Effect of electronic excitation applied by ultrafast lasers on thin film growth A1. Electronically Enhanced Surface Diffusion during Ge Growth on Si(100)

A. Oguz Er and H. E. Elsayed-Ali, J. Appl. Phys. 109, 084320 (6 pages) (2011).

The effect of nanosecond pulsed laser excitation on surface diffusion during growth of $\mathrm{Ge}$ on $\mathrm{Si}(100)$ at $250{ }^{\circ} \mathrm{C}$ was studied. In situ reflection high-energy electron diffraction (RHEED) was used to measure the surface diffusion coefficient while ex situ atomic force microscopy (AFM) was used to probe the structure and morphology of the grown quantum dots. The results show that laser excitation of the substrate increases the surface diffusion during growth of $\mathrm{Ge}$ on $\mathrm{Si}(100)$, changes the growth morphology, improves crystalline structure of the grown quantum dots, and decreases their size distribution. A purely electronic mechanism of enhanced surface diffusion of the deposited Ge is proposed.

\section{A2. Femtosecond pulsed laser deposition of Ge quantum dot growth on $\mathrm{Si}(100)-(2 \times 1)$} A. Oguz Er and H. E. Elsayed-Ali, Appl. Surf. Sci. 257, 8078-8084 (2011).

Ge quantum dots were grown on $\mathrm{Si}(100)-(2 \times 1)$ by pulsed laser deposition at various substrate temperatures using a femtosecond Ti:sapphire laser. In-situ reflection high-energy electron diffraction and ex-situ atomic force microscopy were used to analyze the film structure and morphology. The morphology of germanium islands on silicon was studied at different coverages. The results show that femtosecond pulsed laser deposition reduces the minimum temperature for epitaxial growth of Ge quantum dots to $\sim 280{ }^{\circ} \mathrm{C}$, which is $120^{\circ} \mathrm{C}$ lower than previously observed in nanosecond pulsed laser deposition and more than $200^{\circ} \mathrm{C}$ lower than that reported for molecular beam epitaxy and chemical vapor deposition. 


\section{A3. Low temperature epitaxial growth of Ge quantum dot on $\mathrm{Si}(100)-(2 \times 1)$ by femtosecond laser}

excitation

A. Oguz Er, W. Ren, and H. E. Elsayed-Ali, Appl. Phys. Lett. 98, 013108 (3 pages) (2011); selected for publication in Virtual Journal of Ultrafast Science, February (2011).

Low temperature epitaxy of Ge quantum dots on $\mathrm{Si}(100)-(2 \times 1)$ by femtosecond pulsed laser deposition under femtosecond laser excitation was investigated. Reflection high-energy electron diffraction and atomic force microscopy were used to analyze the growth mode and morphology. Epitaxial growth was achieved at $\sim 70^{\circ} \mathrm{C}$ by using femtosecond laser excitation of the substrate. A purely electronic mechanism of enhanced surface diffusion of the Ge adatoms is proposed.

\section{A4. Excitation induced Ge quantum dot formation on $\mathrm{Si}(\mathbf{1 0 0})-(2 \times 1)$}

A. Oguz Er and H. E. Elsayed-Ali, J. Appl. Phys. 108, 034303 (10 pages) (2010).

The effect of nanosecond pulsed laser excitation on the self-assembly of Ge quantum dots grown by pulsed laser deposition on $\mathrm{Si}(100)-(2 \times 1)$ was studied. In situ reflection high-energy electron diffraction (RHEED) and ex situ atomic force microscopy (AFM) were used to probe the quantum dot structure and morphology. At room temperature, applying the excitation laser decreased the surface roughness of the grown Ge film. With surface electronic excitation, crystalline Ge quantum dots were formed at $250{ }^{\circ} \mathrm{C}$, a temperature too low for their formation without excitation. At a substrate temperature of $390^{\circ} \mathrm{C}$, electronic excitation during growth was found to improve the quantum dot crystalline quality, change their morphology, and decrease their size distribution almost by half. A purely electronic mechanism of enhanced surface hopping of the Ge adatoms is proposed.

\section{A5. Low-temperature growth of InN on $\mathrm{Si}(100)$ by femtosecond pulsed laser deposition}

M. A. Hafez and H. E. Elsayed-Ali, J. Vac. Sci. \& Technol. A 27(4), 696-699 (2009). Also, selected for publication in the Virtual Journal of Ultrafast Science 8 (7) (2009).

InN films were grown on $\mathrm{Si}(100)$ using femtosecond pulsed laser deposition. Laser induced breakdown of ammonia was used to generate atomic nitrogen for $\operatorname{InN}$ growth. An indium buffer layer was initially deposited on the Si substrate at low temperature followed by an InN intermediate layer. The crystal quality and surface morphology were investigated by reflection high-energy electron diffraction during growth and atomic force microscopy and x-ray diffraction after growth. The results showed that the $\operatorname{In}(2 \times 1)$ initial buffer layer improved the quality of the $\operatorname{InN}$ film. High quality $\operatorname{InN}$ films were grown at a temperature of $\sim 350{ }^{\circ} \mathrm{C}$.

(B) Ultrafast electron diffraction studies of femtosecond laser excitation of semimetals (Bi and Sn) Advances in ultrafast X-ray and electron diffraction have made it possible to probe the structure of matter as it undergoes excitation and phase transitions initiated by ultrafast laser pulses. In semiconductors, the excitation of valence electrons into the antibonding conduction band weakens its covalent bonding, causing expansion due to increased repulsive interactions among atoms. For free electron metals like aluminum, the lattice is only affected by electron-phonon collisions. For crystals that are stabilized by the Peierls-Jones mechanism, such as for $\mathrm{Bi}$, femtosecond laser excitation shifts the minimum of the potential energy surface towards a non-Pierls distorted state and causes displacive excitation of coherent phonons of the symmetric $A_{1 g}$ mode.

Ultrafast electron diffraction (UED) is used to monitor the structural dynamics in bismuth and antimony nanoparticles initiated by ultrashort laser pulses $\left(800 \mathrm{~nm}, 120 \mathrm{fs}, 1-6 \mathrm{~mJ} / \mathrm{cm}^{2}\right)$. The femtosecond laser pulse energy density was kept below the damage threshold of the sample. Fig. 1 shows the time-resolved electron diffraction setup. The fundamental wavelength $(800 \mathrm{~nm})$ of a $100-\mathrm{fs}$ Ti:sapphire laser is used to heat the sample, while the frequency tripled pulses are used to activate the photocathode of the electron gun. The optical delay line sets the timing between the laser pulse and electron pulse at the sample.

We used UED to probe the lattice response of Bi nanoparticles to femtosecond laser excitation. Figure 2 shows transmission electron microscopy images of the $\mathrm{Bi}$ nanoparticles, their size distribution, and diffraction pattern. Figure 3(a) and (b) show the time-resolved normalized diffraction intensity of the (012) 
and (110) Bragg peaks and the relative change in lattice parameter along the $\langle 012\rangle$ and $\langle 110\rangle$ directions for pump laser fluence of $2.9 \mathrm{~mJ} / \mathrm{cm}^{2}$. From Fig. 2(a), in the $<012>$ direction the lattice contracts $\sim 0.05 \%$ immediately after photoexcitation (first contraction from $0<\mathrm{t}<10$ ps reaching maximum at $\sim 5 \mathrm{ps}$ ). Following this contraction, the lattice expands to $\sim 0.22 \%$. After the lattice reaches its maximum expansion, it contracts again (second contraction for $t>25 \mathrm{ps}$ ) to a certain position. The magnitude of the contraction and expansion is fluence-dependent. More details on this work is given in manuscripts in Appendix A.

FIG. 1. Layout of the ultrafast electron diffraction setup. A 120 fs pulse is generated from Ti:Sapphire chirped pulse amplifier. The system is running at $1 \mathrm{kHz}$. In this setup, the laser pulse is divided into two parts, one is frequency tripled and used to generate photo-electron from photo-activated electron gun (probe beam), the other part is used to excite the sample (pump beam).

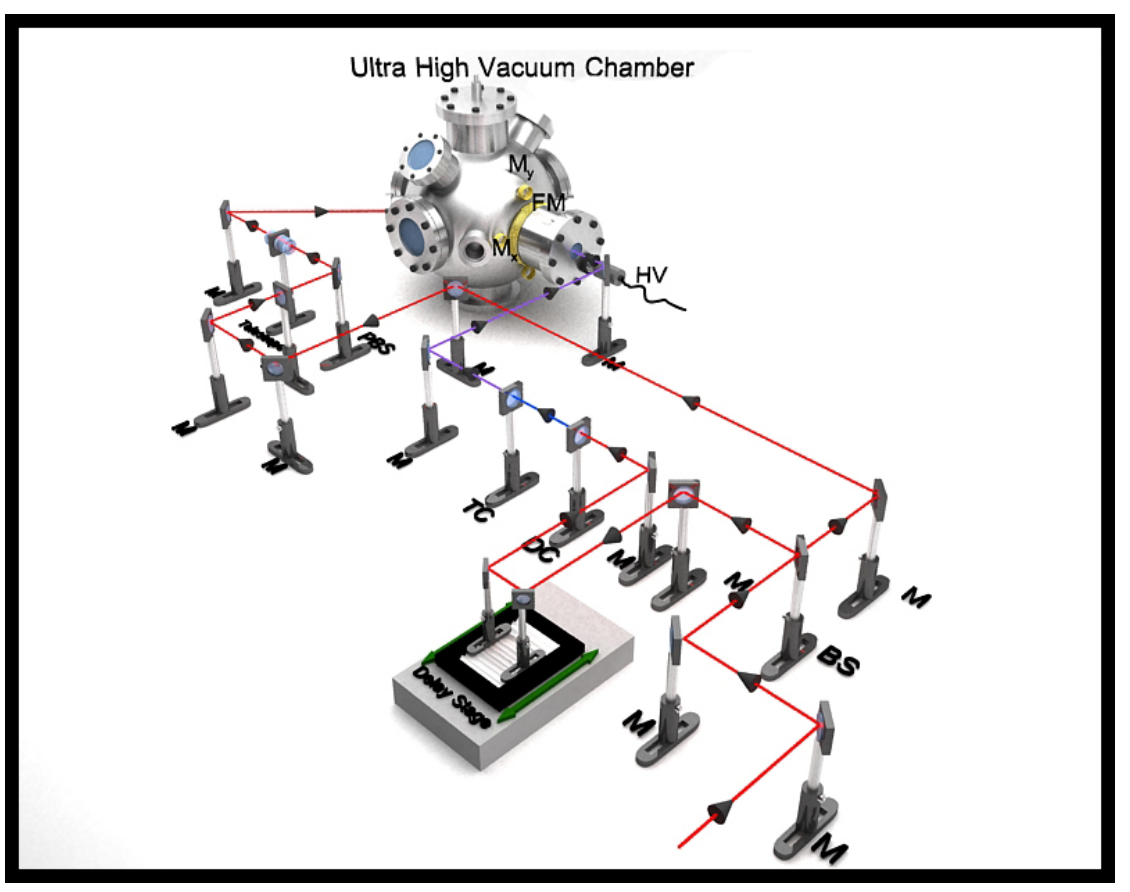

B1. Coherent phonons in bismuth film observed by ultrafast electron diffraction

A. Bugayev, A. Esmail, M. Abdel-Fattah, and H. E. Elsayed-Ali, "Coherent phonons in bismuth film observed by ultrafast electron diffraction," AIP Advances 1, 012117 (2011).

The generation of coherent phonons in polycrystalline bismuth film excited with femtosecond laser pulse is observed by ultrafast time-resolved electron diffraction. The dynamics of the diffracted intensities from the (110), (202), and (024) lattice planes show pronounced oscillations at $130-150 \mathrm{GHz}$. The origin of these coherent acoustic phonons is discussed in view of optical phonon decay into two acoustic phonons. Different drop times in the intensity of the diffraction orders are observed and interpreted as anisotropy in the energy transfer rate of coherent optical phonons.

\section{B2. Anisotropic response of bismuth upon femtosecond laser excitation monitored by ultrafast electron diffraction}

A. R. Esmail and H. E. Elsayed-Ali, "Anisotropic response of bismuth upon femtosecond laser excitation monitored by ultrafast electron diffraction," Appl. Phys. Lett. 99, 161905 (3 pages) (2011).

The lattice response of bismuth 5-nm thin film to femtosecond laser excitation is probed by ultrafast electron diffraction. The transient decay time after laser excitation is longer for diffraction from (012) lattice planes compared to (110) and was reduced with the laser fluence. These results indicate that different energy coupling mechanisms to the lattice occur depending on the crystal direction. The behavior of the diffraction peak width indicates partial disorder of the film upon photoexcitation that increases with the laser fluence. 


\section{B3. Electron diffraction studies of structural dynamics of bismuth nanoparticles}

A. E. Esmail, A. Bugayev, and H. E. Elsayed-Ali J. Phys. Chem. C 117 (17), 9035-9041 (2013).

The lattice response of bismuth nanoparticles to femtosecond laser excitation is probed by ultrafast electron diffraction. Anisotropy in lattice dynamics is detected by monitoring the temporal evolution of the electron diffraction intensity, position, and width for diffraction from the (012) and (110) lattice planes. The transient decay time after laser excitation is observed to be longer for diffraction from (012) lattice planes compared to (110) and was $9.2 \pm 1.1$ ps and $7.5 \pm 1.0$ ps, respectively. By measuring the Bragg angle, a lattice compression is observed followed by expansion along the $<012>$ direction, while along $<110\rangle$ direction only expansion was detected. The behavior of the full width at half maximum of the diffraction peak with time indicates partial disorder of the grown nanoparticles upon photoexcitation.

\section{B4. Nonuniformity in lattice contraction of bismuth nanoclusters heated near its melting point}

A. Esmail, M. Abdel-Fattah, and H. E. Elsayed-Ali, "Nonuniformity in lattice contraction of bismuth nanoclusters heated near its melting point," J. Appl. Phys. 109, 084317 (2011).

The structural properties of $\mathrm{Bi}$ nanoclusters were investigated with transmission electron diffraction from room temperature up to $525 \pm 6 \mathrm{~K}$. The Bi nanoclusters were fabricated by thermal evaporation at room temperature followed by thermal and femtosecond laser annealing. The clusters had an average size of $14 \mathrm{~nm}$ along the minor axis and $\sim 16 \mathrm{~nm}$ along the major axis. The Debye temperature of the annealed nanoclusters was found to be $53 \pm 6 \mathrm{~K}$ along the (012) direction and $86 \pm 9 \mathrm{~K}$ along the (110) direction. At $T=464 \pm 6 \mathrm{~K}$, the diffraction intensity started to deviate from Debye-Waller behavior due to increased lattice anharmonicity. The onset of the melting of the Bi nanoclusters was $T \sim 500 \pm 6 \mathrm{~K}$, as measured by the reduction of the nanocluster size through the formation of a liquid shell detected by the width of the diffraction rings. The thermal expansion coefficient of the $\mathrm{Bi}(012)$ and (110) planes is positive up to $\sim 499$ $\pm 11 \mathrm{~K}$. However, the expansion coefficient of the $\mathrm{Bi}(012)$ planes showed a transition from a positive to a negative value that occurs over the temperature range $T_{c} \sim 499 \pm 11 \mathrm{~K}$ to $511 \pm 8 \mathrm{~K}$. For the $\mathrm{Bi}(110)$ planes, the thermal expansion coefficient is positive up to their melting point, which is $525 \pm 6 \mathrm{~K}$. 